\title{
TWO COUNTABLE HAUSDORFF ALMOST REGULAR SPACES EVERY CONTINUOUS MAP OF WHICH INTO EVERY URYSOHN SPACE IS CONSTANT
}

\author{
V. TZANNES \\ Department of Mathematics \\ University of Patras \\ Patras 26110, Greece
}

(Received July 13, 1990 and in revised form November 2, 1990)

\begin{abstract}
We construct two countable, Hausdorff, almost regular spaces $I(S)$, I (T) having the following properties: (1) Every continuous map of $I(S)$ (resp.I(T)) into every Urysohn space is constant (hence, both spaces are connected). (2) For every point of I(S) (resp.of I(T)) and for every open neighbourhood $U$ of this point there exists an open neighbourhood $V$ of it such that $V \subseteq U$ and every continuous map of $V$ into every Urysohn space is constant (hence both spaces are locally connected). (3) The space $I(S)$ is first countable and the space I(T) nowhere first countable. A consequence of the above is the construction of two countable, (connected) Hausdorff, almost regular spaces with a dispersion point and similar properties. Unfortunately, none of these spaces is Urysohn.
\end{abstract}

KEY WORDS AND PHRASES. Hausdorff, Urysohn, almost regular, connected, locally connected, dispersion point.

1980 AMS SUBJECT CLASSIFICATION CODE. 54D05, 54D10, 54G09 54 G99.

\section{INTRODUCTION.}

Iliadis and Tzannes [1] posed the question whether for every countable Hausdorff space $R$ there exists a countable Hausdorff (Urysohn, almost regular) space $I(X)$ having the following properties:

(1) Every continuous map of $I(X)$ into the given space $R$ is constant

(2) For every point $x$ of $X$ and for every open neighbourhood $U$ of $x$ there exists an open neigbourhood $V$ of $x$ such that $V \subseteq U$ and every continuous map of $V$ into the given space $R$ is constant. (Spaces having properties (1) and (2) are called in [1], R-monolithic and locally R-monolithic, respectively, and by their construction are connected and locally connected).

It is obvious that the above mentioned spaces $I(S), I(T)$ answer partially this question (in case the countable space $R$ is Urysohn) because both have properties (1) and (2) for every Urysohn space.

For countable spaces, first countable or nowhere first countable, connected, locally connected Hausdorff or Urysohn, almost regular or 
having a dispersion point, or with other properties see [1]-[37].

A space $X$ is called 1) Urysohn, if for every two distinct points $x, y$ of $x$ there exist open neighbourhoods $V$, $U$ of the points $x, y$ such that $\bar{v} n \bar{U}=\varnothing$ 2) Almost regular if it has a dense subset of regular points. A point $p$ of a space $x$ is called a regular point cor, that $x$ is regular at $p$ ) if for every open neighbourhood $U$ of $p$ there exists an open neighbourhood $v$ of $p$ such that $\bar{v} \subseteq U$.

A point $p$ of a connected space $X$ is called a dispersion point if the space $x \backslash\{p\}$ is totally disconnected.

Let $X$ be a set and let $\left\{X_{1}: i \in I\right\}$ be a family of subsets of $x$ with each $x_{i}$ having a topology. Assume that for every $(i, j) \in I \times I$ both 1$)$ The topologies of $x_{1}, x_{j}$, agree on $\left.x_{2} n x_{3}, 2\right)$ Each $x_{2} n x_{3}$ is open in $x_{2}$ and in $x_{j}$. Then the weak topology in $X$ induced by $\left\{X_{1}: i \in I\right\}$ is $\tau=\left\{U: U n X_{2}\right.$ is open in $x_{1}$ for every $i \in I$ ?.

2. AN AUXILIARY SPACE.

The following space $X$ which is due to Urysohn [35], will be used for the construction of two auxiliary spaces $S, T$ which with the help of the embedding described in [1] will yield the required spaces.

On the set $x=\left\{a_{i j}, b_{1,}, c_{1}, a, b: i=1,2, \ldots, j=1,2, \ldots,\right\}$ we define the following topology: Each $a_{i j}, b_{i j}, i=1,2, \ldots, j=1,2, \ldots$ is isolated. A basis of open neigbourhoods of the points $c_{i}, i=1,2, \ldots, a, b$ are the sets

$$
\begin{aligned}
& B\left(c_{i}\right)=\{V^{n}\left(c_{i}\right)=\underbrace{\infty}_{j=n}\left\langle a_{1 j}, b_{1 j}, c_{1}\right\}: n=1,2, \ldots,\} \\
& B(a)=\left\{v^{n}(a)=\bigcup_{j=1}^{\infty} j \bigcup_{1=n}^{\infty}\left\langle a_{1 j}, a\right): n=1,2, \ldots\right\} \\
& B(b)=\left\{V^{n}(b)=\bigcup_{j=1}^{\infty} \int \bigcup_{1=n}^{\infty} J\left(b_{1 j}, b\right): n=1,2 \ldots,\right\}
\end{aligned}
$$

The countable space $X$ has the following properties:

(1) It is Hausdorff, almost regular (all points of $x$ besides $a, b$ are regular points).

(2) $f(a)=f(b)$, for every continuous map $f$ of $x$ into every Urysohn space, (because the points $a, b$ can not be separated by disjoint closed neighbourhoods).

Let $x_{n}, n=1,2, \ldots$ be disjoint copies of $x$ and let $a_{n}, b_{n}$ be the copies of $a, b$, respectively in the space $x_{n}$.

For every $n=1,2, \ldots$ we attach the space $x_{n}$ to the space $x_{n+1}$ identifying the point $b_{n}$ with $a_{n+1}$. We set $a_{1}=x_{0}, b_{n}=a_{n+1}=x_{n}, n=1,2, \ldots$ and on the space

$$
\left.Y=\left(x_{0}, x_{1}, \ldots, x_{n} \ldots\right) \cup \bigcup_{n=1}^{\infty}\right)\left(x_{n} \backslash\left(a_{n}, b_{n}\right)\right.
$$

we add one more point $p$.

On the set $Z=Y$ K $p$ we define the basis of open neighbourhoods of the point $p$ to be the sets 


$$
U_{n}(p)=\left(x_{1}: i \geq n\right) U \bigcup_{1=n+1}^{\infty} J\left(x \backslash a_{n} \cdot b_{n},\right) \bigcup U\left(x_{n}\right)
$$

$n=1,2, \ldots$, where $U\left(x_{n}\right)$ is an open set of $x_{n}$ in $x_{n}$. Since

$$
\overline{U_{n+1}(p)}=\left\{x_{1}: i \geq n+1\right\} \cup\left(\sum_{1=n+2}^{\infty} J\left(x_{n+1} \backslash\left(a_{n+1}, b_{n+1}\right)\right) \bigcup J\left(x_{n+1}\right)\right.
$$

it follows that $\overline{U_{n+1}(p)} \subseteq U_{n}(p)$, that is, $Z$ is regular at the point $p$. Now consider two disjoint copies $z^{-}, z^{+}$of $z$ and let $x_{n}^{-}, x_{n}^{+}$be the copies of $x_{n}$ in $z^{-}, z^{+}$, respectively. Let $x_{0}^{-} x_{0}^{+}$and $p^{-}, p^{+}$be the copies of $x_{0}$ and $p$ in $z^{-}, z^{+}$, respectively. We attach the copy $z^{-}$to $z^{+}$ identifying $x_{0}^{-}$with $x_{0}^{+}$. We set $x_{0}^{-}=x_{0}^{+}=0$ and we consider the space

$S=\left(Z^{-} \backslash\left(x_{0}^{-}\right) \cup J 0\right) \bigcup\left(Z^{+} \backslash\left(x_{0}^{+}\right\}\right)$which has the following properties:

(1) It is Hausdorff almost regular (all points of $S$ besides $\left(x_{n}^{-}, 0, x_{n}^{+}: n=1,2, \ldots,\right\}$ are regular points $)$.

(2) $f\left(p^{-}\right)=f\left(p^{+}\right)$, for every continuous map $f$ of $S$ into every Urysohn space. (To prove this observe that since $S$ is not Urysohn at every pair $\left(x_{n+1}^{-}, x_{n}^{-}\right),\left(x_{1}^{-}, 0\right),\left(0, x_{1}^{+}\right),\left(x_{n}^{+}, x_{n+1}^{+}\right), n=1,2, \ldots$ it follows that $f\left(x_{n+1}^{-}\right)=f\left(x_{n}^{-}\right)=f(0)=f\left(x_{n}^{+}\right)=f\left(x_{n+1}^{+}\right)$. for every $n=1,2, \ldots$ and hence $f\left(p^{-}\right)=f\left(p^{+}\right)$for every continuous map $f$ of $S$ into every Urysohn space).

3. MAIN RESULTS.

PROPOSITION 3.1. There exists a countable, first countable, Hausdorff, almost regular space $I(S)$ having the following properties:

(1) Every continuous map of $I(S)$ into every Urysohn space is constant (hence $I(S)$ is connected).

(2) For every point $s$ of $I(S)$ and for every open neighbourhood $U$ of $s$, there exists an open neighbourhood $V$ of $s$, such that $V \subseteq U$ and every continuous map of $V$ into every Urysohn space is constant (hence $I(S)$ is locally connected).

PROOF. Let $S$ be the space consrtucted above. We set $J=S \backslash\left(\mathrm{p}^{-}, \mathrm{p}^{+}\right\}$, $\Lambda_{0}=S x S \backslash \Delta(S) \quad \Delta(S)=\{(x, y) \in S x S: x=y\}$ and we construct, as in [1], first the space $I^{1}\left(S, \Lambda_{0}\right)=S \bigcup \underbrace{}_{\lambda \in \Lambda_{0}} J^{\lambda}$, then, by induction, the space $I^{n}\left(S, \Lambda_{n-1}\right)=I^{1}\left(I^{n-1}\left(S, \Lambda_{n-2}\right), \Lambda_{n-1}\right)$ and finally, the space $I(S)=\bigcup_{n=1}^{\infty} J I^{n}\left(S, \Lambda_{n-1}\right)$.

That $I(S)$ is Hausdorff almost regular is proved as in [1, Lemma 2 ].

That it is first countable follows by the fact that $S$ is first countable (all spaces $X, Y, Z$ are first countable) and by relation ( 7 ) [1, Theorem 1] which in this case becomes $x(I(S))=\max \left(x(S), N_{0}\right)=N_{0}$.

In order to prove Properties (1) and (2), observe that by the property of the space $S$ (that $f\left(p^{-}\right)=f\left(p^{+}\right)$, for every continuous map of $S$ into every Urysohn space) and by the definition of topology on $I^{n}\left(S, \Lambda_{n-1}\right)$ it follows that 1$)$ Every continuous map of $I^{n}\left(S, \Lambda_{n-1}\right)$ into every Urysohn space is constant on $I^{n-1}\left(S, \Lambda_{n-2}\right)$ and 2$)$ For every point 
$s$ of $I^{n-1}\left(S, \Lambda_{n-2}\right)$ and for every open neighbourhoid $U$ of $s$ in $I^{n}\left(S, \Lambda_{n-1}\right)$, there exists an open neighbourhood $V$ of $s$ in $I^{n}\left(S, \Lambda_{n-1}\right)$ such that $V \subseteq U$ and every continuous map of $V$ into every Urysohn space, is constant on $V \cap I^{n-1}\left(S, \Lambda_{n-2}\right)$. Finally, by the definition of topology on $I(S)$ it follows that 1 ) Every continuous map of $I(S)$ into every Urysohn space is constant (hence $I(S)$ is connected because the set of real-numbers with the usual topology is a Urysohn space) and 2) For every point $s$ of $I(S)$ and for every open neighbourhood $U$ of $s$ there exists an open neighboorhood $V$ of $s$ such that $V \subseteq U$ and every continuous map of $V$ into every Urysohn space is constant (hence I(S) is locally connected).

REMARK 3.1. If we consider as initial space the space $x$ of Section 1 then the resulting space $I(X)$ will be a countable, first countable. Hausdorff, anti-Urysohn space having Properties (1) and ( 2 ). (a space $S$ is called anti-Urysohn if for every $x, y \in S$ and for every open neighbourhoods $U, V$ of $x, y$ respectively, Ün $\bar{x} \times \theta$ ).

REMARK 3.2. If on the set $I^{1}\left(S, \Lambda_{0}\right)$ we define the topology to be the weak topology induced by the spaces $J^{\lambda}, A \in \Lambda_{0}$ and $S$, then the space $I^{1}\left(S, \Lambda_{0}\right)$ is not first countable at every point of $S$. Hence the set $I^{n}\left(S, \Lambda_{n-1}\right)$ with the weak topology induced by the spaces $J^{\lambda}$, $\lambda \in \Lambda_{n-1}$ and $I^{n-1}\left(S, \Lambda_{n-2}^{n-1}\right)$ is not first countable at every point of $I^{n-1}\left(S, \Lambda_{n-2}\right)$.

Therefore if on the set $I(S)=\bigcup_{n=1}^{\infty} I^{n}\left(S, \Lambda_{n-1}\right)$ we define the topology to be the weak topology induced by the spaces $I^{n}\left(S, \Lambda_{n-1}\right), n=1,2, \ldots$, then the space I(S) will be nowhere first countable. It is easy to prove that while I(S) is Hausdorff almost regular having Property ( 1 ), it is not locally connected (hence does not have Property (2)).

PROPOSITION 3.2. There exists a countable, nowhere first countable, Hausdorff, almost regular space having properties (1) and (2) of Proposition 3.1.

PROOF. We consider the space $M=\mathbb{N} \bigcup\{p\}$, $p \in B N \backslash \mathbb{N}$, where $\mathbb{N}$ is the set of natural numbers and $\beta N$ is the Stone-Cech compactification of $\mathbb{N}$. The space $M$ is countable regular and not first countable at the point $p$.

Let $M_{1}, M_{2}$ be two disjoint copies of $M$ and let $p_{1}, P_{2}$ be the copies of $p$ in $M_{1}, M_{2}$, respectively. We attach the copies $M_{1}, M_{2}$ to the space $S$ attaching the point $p_{1}$ to $p^{-}$and the point $p_{2}$ to $p^{+}$. We consider the space $T=S \bigcup M_{1}^{\prime} \bigcup M_{2}^{\prime}$, where $M_{1}^{\prime}=M_{1} \backslash\left(p_{1}\right), i=1,2$.

Obviously, the space $T$ is Hausdorff almost regular, not first countable at the points $p^{-}, p^{+}$and $f\left(p^{-}\right)=f\left(p^{+}\right)$, for every continuous map $f$ into every Urysohn space.

The space I(T) constructed as in Proposition 3.1 is the required space.

COROLLARY 3.1. There exists a countable, first countable, cor nowhere first countable) Hausdor $f$, almost regular space having the following properties:

(1) Every continuous map of it into every Urysohn space is constant 
(hence it is connected).

(2) It has a dispersion point.

(3) For every open neighbourhood $U$ of the dispersion point there exists an open neighbourhood $V$ of 1 t such that every continuous map of $V$ into every Urysohn space is constant (hence it is locally connected only at the dispersion point).

PROOF. First we observe that both spaces $S$ and $T$ of Section 2 and Proposition 3.3, respectively are total ly disconnected. Hence, we can apply [1. Theorem 2] using as initial space the space $S$, for the construction of countable first countable and the space $T$, for the construction of the countable nowhere first countable space. The other properties of both spaces are proved as in Proposition 3.1 .

\section{REFERENCES}

1. ILIADIS, S. and TZANNES, V. . Spaces on which every continuous map into a given space is constant, Canad. J.Math. 38 (1986), 12811298.

2. ANDERSON, D.R., On connected irresolvable Hausdorff spaces, Proc. Amer. Math. Soc. 12 (1961), 165-167.

3. BAGLEY, R.W., Another way of constructing countable connected Hausdorff spaces, Math. Japon. 30 (2) (1985), 235-237.

4. BAGGS, I., A connected Hausdorff space which is not contained in a maximal connected space, Pacific J.Math. 51 (1974), 11-18.

5. BING, R.H., A connected countable Hausdorff space, Proc. Amer. Math. Soc. 4 (1953), 474.

6. BOYLES, S.M. and RITTER, G.X., A connected locally connected countable space which is almost regular, Colloq. Math. 46 (1982), 189-195.

7. BROWN, M., A countable connected Hausdorff space, Bull. Amer. Math. Soc. 59 (1953), 367.

8. CVID, S.F., Countable connected spaces, Current Questions in Mathematical Logic and Set Theory (in Russian), Moskov. Gos. Ped. Inst. im. Lenina, Moscow 1975, 276-284.

9. CVID, S.F., A countable strongly unicoherent space (in Russian), Mat. Zametki 24 (1978), 289-294 (English translation: Math. Notes 24(1978), 655-657).

10. GOLOMB, S.W., A connected topology for the integers, Amer. Math. Monthly 66 (1959), 663-665.

11. GUSTIN, W., Countable connected spaces. Bull. Amer. Math. Soc. 52 (1946), 101-106.

12. HARLEY, P.W. A countable nowhere first countable Hausdorff space, Canad. Math. Bull. 16 (1973), 441-442.

13. HEWTT, E., On two problems of Urysohn, Ann. of Math. 47 (1946), 503-509.

14. JONES, F.B., and STONES, A.H., Countable locally connected Urysohn spaces, Colloq. Math. 22 (1971), $239-244$.

15. KANNAN, V., On three questions of Arhangel'skii and Franklin, Not. Amer. Math. Soc. 17 (1970), 839-840, 70T-G123.

16. KANNAN, V., A countable connected Urysohn space containing a dispersion point, Proc. Amer. Math. Soc. 35 (1972), 289-290.

17. KANNAN, V., and RAJAGOPALAN, M., Regularity and dispersion in countablespaces, Duke Math. J. 39 (1972), 729-734. 
18. KANNAN, V., On rountable locally connerted spaces, Colloy. Math. 29 (1974), 93-100.

19. KIRCH, A. M. A countable, connected, locally connected Housdorff space. Amer. Math. Monthly 76 (1969),169-171.

20. LARMORE, L., A connected countable Hausdorff space Sa for every countable ordinal $\alpha$. Bol. Soc. Mat. Mexicana (2) 17 (1972), 14-17.

21. LEVY, R., Countable spaces without points of first countability. Pacific J. Math. 70 (2) (1977), 391-399.

22. MARTIN, J., A countable Hausdorff space with a dispersion point, Duke Math. J. 33 (1966), 165-167.

23. MICHAELIDES, G. J., On countable connected Hausdorff spaces, Studies and Essays. Mathematics Research Center. National Taiwan University Taipei. Taiwan, China (October 1970), 183-189.

24. MILLER, G.G., Countable connected spaces, Proc. Amer. Math. Soc. 26 (1970), 355-360.

25. MILLER, G.G., A countable Urysohn space with an explosion point, Notices Amer. Math. Soc. 13 (1966), 589.

26. MILLER, G.G. A countable locally connected quasimetric space, ibldem 14 (1967), 720 .

27. MILLER,G.G. and PEARSON B.J., On the connectification of a space by a countable point set, J.Austral. Math. Soc. 13 (1972), 67-70.

28. ORI, R.G. and RAGAGOPALAN, M., On countable connected locally connected almost regular Urysohn spaces, Gen. Top. Appl. 17 (1984), 157-171.

29. RITTER, G.X., A connected, locally connected, countable Hausdorff space, Amer. Math. Monthly 83 (1976), 185-186.

30. RITTER, G.X., A connected, locally connected, countable Urysohn space, Gen. Top. Appl. 7 (1977), 65-70.

31. ROY, P., A countable connected Urysohn space with a dispersion dispersion point, Duke Math. J. 33 (1966), 331-334.

32. SHIMRAT, M. Embedding in homogeneous spaces, Quart. J. Math. (2) 5 (1954), 304-311.

33. STONE, A.H., A countable connected, locally connected Hausdorff Hausdorff space. Notices Amer. Math. Soc. 16 (1969), 442.

34. TZANNES, V. Three countable connected spaces, Colloq. Math. 2 (1988), 267-279.

35. URYSOHN, P., Uber die Machtigkeit der zusammenhangenden Mengen, Math. Ann. 94 (1925), 262-295.

36. VOUGHT, E.J., A countable connected Urysohn space with a dispersion point that is regular almost everywhere. Colloq. Math. 28 (1973), 205-209.

37. WILLMOTT, R., Countable yet nowhere first countable, Math. Mag. $\underline{52}$ (1) $(1979), 26-27$. 


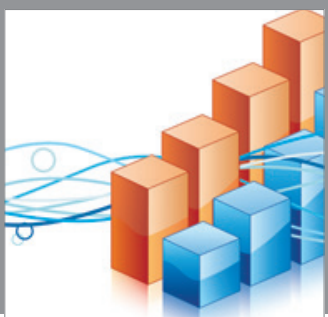

Advances in

Operations Research

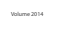

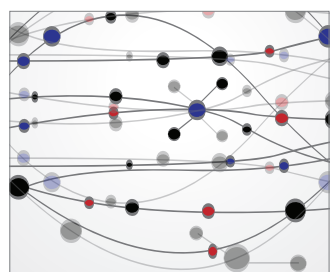

\section{The Scientific} World Journal
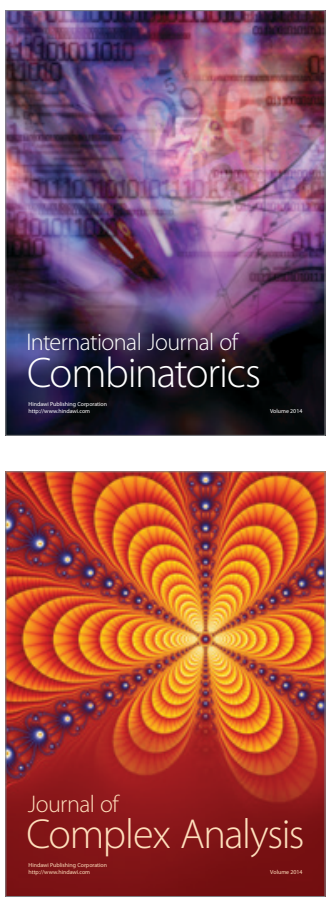

International Journal of

Mathematics and

Mathematical

Sciences
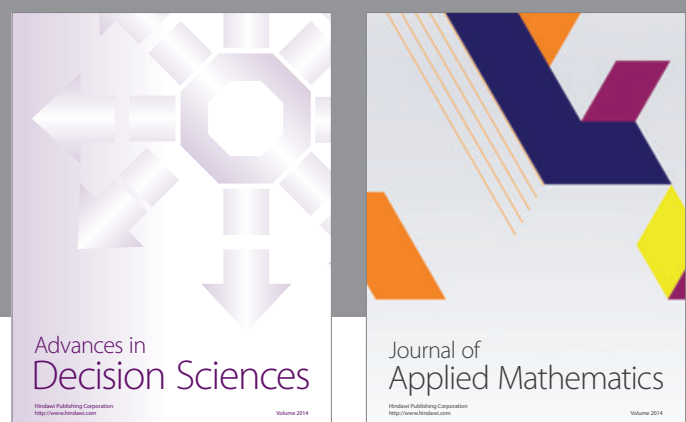

Journal of

Applied Mathematics
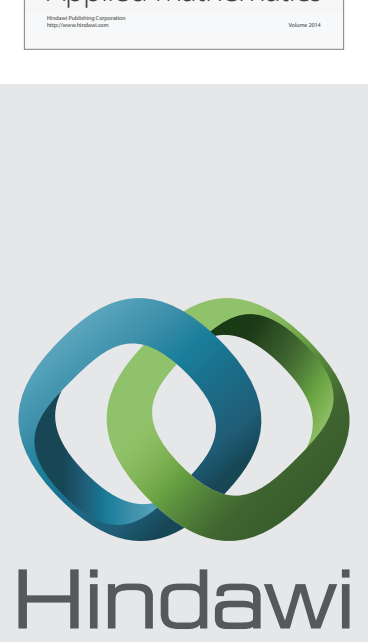

Submit your manuscripts at http://www.hindawi.com
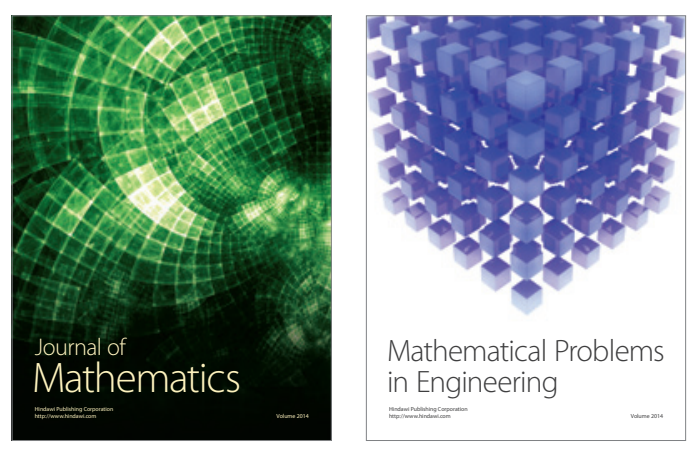

Mathematical Problems in Engineering
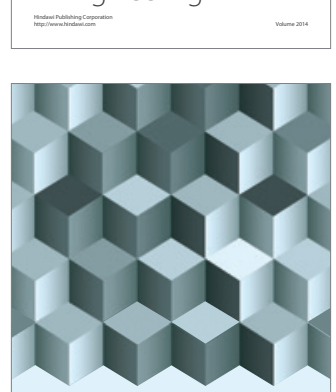

Journal of

Function Spaces
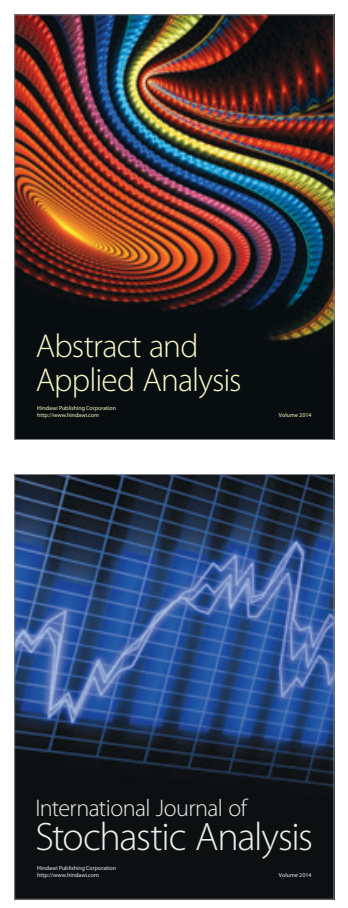

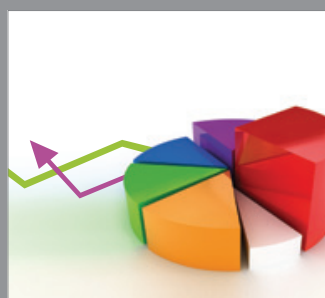

ournal of

Probability and Statistics

Promensencen
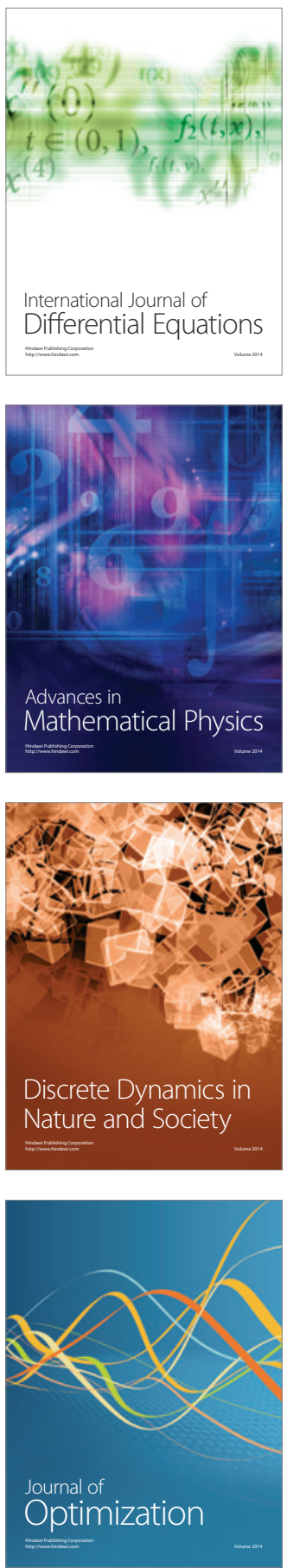